\title{
OPTIMAL INVESTMENT STRATEGY FOR A NON-LIFE INSURANCE COMPANY: QUADRATIC LOSS
}

Abstract. The aim of this paper is to construct an optimal investment strategy for a non-life insurance business. We consider an insurance company which provides, in exchange for a single premium, full coverage to a portfolio of risks which generates losses according to a compound Poisson process. The insurer invests the premium and trades continuously on the financial market which consists of one risk-free asset and $n$ risky assets (Black-Scholes market). We deal with the insurer's wealth path dependent disutility optimization problem and apply a quadratic loss function which penalizes deviations below a reserve for outstanding liabilities as well as above a given upper barrier. An optimal investment strategy is derived using stochastic control theory in the absence of constraints on control variables. Some properties of the strategy and the behaviour of the insurer's wealth under the optimal control are investigated. The set up of our model is more general, as it can also be used in non-life loss reserving problems.

1. Introduction. In the past few years optimal portfolio selection problems have gained much interest in financial and actuarial literature. There are at least two reasons for this: the diversity of investment possibilities on financial markets and new regulatory requirements.

The idea of portfolio selection is to construct the best allocation of wealth among assets in order to finance a future stream of spending over a given time horizon. This allocation of wealth changes dynamically over time, which implies rebalancing of the investment portfolio.

In this paper we investigate the problem of identifying the optimal investment strategy for a non-life insurance company. Portfolio selection problems

2000 Mathematics Subject Classification: 91B28, 91B30, 93E20.

Key words and phrases: optimal investment strategy, insurer's wealth path dependent disutility optimization, Hamilton-Jacobi-Bellman equation, Lévy-type stochastic integrals. 
for non-life insurance business are rather rare in actuarial literature. Stochastic control theory is usually applied to non-life insurance models in order to find an optimal dividend payout scheme or optimal reinsurance contract (see for example Hipp and Vogt (2003), Højgaard and Taksar (2002), Hubalak and Schachermayer (2004), Paulsen (2003) and Schmidli (2002)). For a survey of control theory in ruin theory models see also Hipp (2002) who deals with optimal investment, optimal proportional reinsurance, optimal XL reinsurance, optimal premium control and optimal new business.

Most portfolio selection problems in actuarial mathematics concern optimal asset allocations for defined benefit or defined contribution pension plans and other retirement problems (see for example Cairns (2000) and Haberman and Vigna (2002)). This is due to the fact that pension plans are long-term contracts and investment uncertainty is greater than for short-term non-life contracts. However, the financial market offers the possibility to improve company's financial position and a non-life insurer should be interested in trading assets on the market even in short-term.

As far as investment choices of a non-life insurance company on a stock market are concerned, there are two prominent papers of Browne (1995) and Hipp and Plum (2000). Browne approximates an insurance surplus process by a Brownian motion with a drift and determines an investment strategy which maximizes the expected exponential utility from final wealth. It is shown that this strategy also minimizes the ruin probability. Hipp and Plum (2000) derive an investment strategy which minimizes the ruin probability in a classical collective risk model with infinite time horizon. The recent paper of Korn (2005) extends the result of Browne (1995) by introducing the concept of worst-case portfolio optimization (which takes into account the possibility of a market crash) and by solving the maximization problem of worst-case exponential utility from final wealth.

We consider an insurance company which collects a single premium, invests it and trades continuously on the financial market adopting an investment strategy which is optimal in the sense of a specified loss function. We investigate the wealth path dependent disutility optimization problem as the insurer's performance is evaluated in continuous time during the whole term of the policy. Applying an optimal investment strategy, the insurer should meet all future unpredictable liabilities. It seems that our wealth path dependent disutility optimization approach to the investment problem of a non-life insurer is a novelty as most papers deal with maximization of the utility from final wealth or minimization of the ruin probability. If we take a single premium to be an amount of money set aside by the insurer for the future unpaid liabilities, we obtain an investment problem for non-life loss reserving. For non-life reserving problems in the context of portfolio selection see Ahcan et al. (2004). 
This paper is structured as follows. In Section 2 we set up a non-life insurance contract and the financial market, and state our optimization problem. In Section 3 we derive an optimal investment strategy for the problem without constraints on control variables. Section 4 deals with the properties of the strategy and the behaviour of the insurer's wealth under the optimal control.

2. The general model. In this section we introduce an optimization model for an insurance company which issues a non-life policy and trades assets on a financial market.

We are given a probability space $(\Omega, \mathcal{F}, \mathbb{P})$ with filtration $\mathbb{F}=\left(\mathcal{F}_{t}\right)_{0 \leq t \leq T}$ and $\mathcal{F}_{T}=\mathcal{F}$, where $T$ is a time horizon. The filtration satisfies the usual hypotheses of completeness $\left(\mathcal{F}_{0}\right.$ contains all sets of $\mathbb{P}$-measure zero $)$ and right continuity $\left(\mathcal{F}_{t}=\mathcal{F}_{t+}\right)$.

Consider the collective risk model. Let $J(t)$ denote the aggregate claim amount up to time $t$, for $0 \leq t \leq T$. We assume that the process $\{J(t), 0 \leq$ $t \leq T\}$ is a compound Poisson process, which means that it has a representation

$$
J(t)=\sum_{i=1}^{N(t)} Y_{i},
$$

where $\left\{Y_{i}, i \in \mathbb{N}\right\}$ is a sequence of positive, independent and identically distributed random variables with law $p$ and $N(t)$ is a homogeneous Poisson process with intensity $\lambda$ which is independent of the sequence $\left\{Y_{i}, i \in \mathbb{N}\right\}$. We assume that the process $\{J(t), 0 \leq t \leq T\}$ is $\mathbb{F}$-adapted with càdlàg sample paths (continuous on the right and having limits on the left). In insurance risk models, $Y_{1}, Y_{2}, \ldots$ denote the amounts of successive claims and $N(t)$ counts the number of claims up to time $t$. The probability measure $p$ satisfies $\int_{0}^{\infty} y^{4} p(d y)<\infty$, which means that the random variables $\left\{Y_{i}, i \in \mathbb{N}\right\}$ have first four moments finite; let $\mu=\mathbb{E}\left(Y_{i}\right)$ and $\mu_{2}=\mathbb{E}\left(Y_{i}^{2}\right)$. Let $T_{i}=\inf \{t \geq 0$ : $N(t)=i\}$, with $T_{0}=0$, denote the time of the $i$ th claim and $\xi_{i}=T_{i}-T_{i-1}$ the inter-claim time. It is well known that $\left\{\xi_{i}, i \in \mathbb{N}\right\}$ is a sequence of independent and exponentially distributed random variables which are independent of the sequence $\left\{Y_{i}, i \in \mathbb{N}\right\}$.

The compound Poisson process $\{J(t), 0 \leq t \leq T\}$ can also be defined through the Poisson stochastic integral of the form

$$
J(t)=\int_{0}^{t} \int_{0}^{\infty} z M(d s, d z),
$$

where $M(t, A)=\#\{0 \leq s \leq t: \triangle J(t) \in A\}, \triangle J(t)=J(t)-J(t-)$, for all Borel sets $A \in \mathcal{B}(0 ; \infty)$, is a Poisson random measure with intensity measure $\lambda p(A)$. The random measure $M(t, A)$ counts the jumps of the compound 
Poisson process of a specified size of $A$ up to time $t$ and has Poisson distribution. For more details on Poisson random measures, Poisson stochastic integrals and general Lévy-type stochastic integrals see Applebaum (2004).

On a financial market, there are $n+1$ financial assets. One of the assets is risk-free and its price $\{B(t), 0 \leq t \leq T\}$ is described by an ordinary differential equation of the form

$$
\frac{d B(t)}{B(t)}=r d t, \quad B(0)=1
$$

where $r$ denotes the force of interest. The remaining assets are risky and their prices $\left\{S_{i}(t), 0 \leq t \leq T\right\}$, for $i=1, \ldots, n$, evolve according to stochastic differential equations

$$
\frac{d S_{i}(t)}{S_{i}(t)}=a_{i} d t+\sum_{j=1}^{n} \sigma_{i j} d W_{j}(t), \quad S_{i}(0)>0,
$$

where $a_{i}$ denotes the expected return on the risky asset $i, \sigma_{i j}$ denote deterministic volatilities, for $j=1, \ldots, n$, and $\left\{W_{1}(t), \ldots, W_{n}(t), 0 \leq t \leq T\right\}$ is a standard $n$-dimensional Brownian motion, $\mathbb{F}$-adapted, which is independent of the compound Poisson process $J(t)$.

Let us consider a portfolio of insurance risks which generates losses according to the compound Poisson process. An insurance company issues a policy, with the term of $T$ years, covering all claims from that portfolio which occur during the term of the contract. In exchange for that protection, the insurance company receives a collective, single premium in the amount of $x_{0}$, invests the premium on the financial market and trades assets continuously in order to improve its financial position. At each time, the insurer adopts an investment strategy $\left(\theta_{1}(t), \ldots, \theta_{n}(t)\right)$, where $\theta_{i}(t)$ is the fraction of the available wealth invested in the risky asset (stock) $i$ at time $t$. The remaining fraction of the wealth, $\theta_{0}(t)=1-\sum_{i=1}^{n} \theta_{i}(t)$, is invested in the risk-free asset (bank account). We assume that the adopted strategy $\left\{\theta_{1}(t), \ldots, \theta_{n}(t), 0<t \leq T\right\}$ is a predictable process with respect to the filtration $\mathbb{F}$.

Let $X(t)$, for $0 \leq t \leq T$, denote the value of the insurer's wealth arising from the insurance contract. In the classical risk theory, the process $\{X(t), 0 \leq t \leq T\}$ is called the insurance surplus process. It evolves according to the stochastic differential equation

$$
\begin{aligned}
d X(t)= & \sum_{i=1}^{n} \theta_{i}(t) X(t-)\left\{a_{i} d t+\sum_{j=1}^{n} \sigma_{i j} d W_{j}(t)\right\} \\
& +\left(1-\sum_{i=1}^{n} \theta_{i}(t)\right) X(t-) r d t-d J(t), \quad X(0)=x_{0}
\end{aligned}
$$


We require that investment strategies satisfy the conditions

$$
\begin{gathered}
\mathbb{P}\left(\int_{0}^{T} \theta_{i}(t)^{2} X(t-)^{2} d t<\infty\right)=1, \quad i=1, \ldots, n, \\
\mathbb{P}\left(\int_{0}^{T} \theta_{0}(t) X(t-) d t<\infty\right)=1 .
\end{gathered}
$$

These conditions ensure that the stochastic differential equation (2.5) is well defined and that the process $\{X(t), 0 \leq t \leq T\}$ is an $\mathbb{F}$-adapted semimartingale with càdlàg sample paths. Strategies which are predictable processes with respect to the filtration $\mathbb{F}$ and satisfy (2.6) and (2.7) are called admissible for our problem.

The above stochastic differential equation can be rewritten in matrix form as

$$
d X(t)=X(t-) \theta(t)^{T} \pi d t+X(t-) \theta(t)^{T} \Sigma d \mathbb{W}(t)+X(t-) r d t-d J(t),
$$

with $X(0)=x_{0}$, where $\theta(t)^{T}=\left(\theta_{1}(t), \ldots, \theta_{n}(t)\right)$ denotes the investment strategy adopted by the insurer, $\pi^{T}=\left(\pi_{1}, \ldots, \pi_{n}\right)$ the vector of risk premiums attached to given assets $\left(\pi_{i}=a_{i}-r\right), \Sigma$ the matrix of volatilities and $\mathbb{W}(t)^{T}=\left(W_{1}(t), \ldots, W_{n}(t)\right)$ the standard $n$-dimensional Brownian motion. A natural assumption is to consider positive risk premiums. We assume that the matrix of volatilities is nonsingular. This implies that the covariance matrix $\mathbb{Q}=\Sigma \Sigma^{T}$ is positive definite. It is worth noting that the process $\{X(t), 0 \leq t \leq T\}$ is a Lévy-type stochastic integral as its stochastic differential can be represented in the form

$$
\begin{aligned}
d X(t)= & \left(X(t-) \theta(t)^{T} \pi+X(t-) r-\int_{0<y<1} y \lambda p(d y)\right) d t \\
& +X(t-) \theta(t)^{T} \Sigma d \mathbb{W}(t)-\int_{y \geq 1} y M(d t, d y) \\
& -\int_{0<y<1} y(M(d t, d y)-\lambda p(d y) d t), \quad X(0)=x_{0} .
\end{aligned}
$$

During the term of the policy, the insurance company sets aside a reserve. The reserve is the amount of money which should ensure that the insurer will meet all the future contractual obligations arising from the portfolio. The reserve is usually calculated under prudential basis. This means that the insurer assumes in the calculations a higher loss frequency and a more severe claim size distribution.

Traditionally, actuaries calculate reserves for the outstanding liabilities as the conditional expected value of all future discounted payments, 


$$
R(t)=\mathbb{E}\left[\int_{t}^{T} e^{-\widehat{\delta}(s-t)} d \widehat{J}(s) \mid \mathcal{F}_{t}\right], \quad 0 \leq t<T,
$$

where $\widehat{\delta}$ is a prudent rate of return on investments (discount rate) and $\widehat{J}(t)$ is a compound Poisson process with Poisson intensity $\widehat{\lambda} \geq \lambda$ and claim size distribution with finite first moment $\widehat{\mu} \geq \mu$.

In order to calculate the above expected value we use the following well known fact from the theory of Poisson processes (see for example Sato (1999)).

LEMma 2.1. The distribution of the random vector $\left(T_{1}, \ldots, T_{n}\right)$, conditioned on $N(t)=n$, is given by the density function

$$
f_{\left(T_{1}, \ldots T_{n}\right) \mid N(t)=n}\left(t_{1}, \ldots, t_{n}\right)=\frac{n !}{t^{n}}, \quad 0 \leq t_{1} \leq \cdots \leq t_{n} \leq t,
$$

which means that the random vector $\left(T_{1}, \ldots, T_{n}\right)$, conditioned on $N(t)=n$, is distributed as the vector of $n$ ordered statistics taken from the uniform distribution on the interval $[0, t]$.

With the above lemma, calculation of the expectation in (2.10) is straightforward. Because of the independence and stationarity of the increments of the compound Poisson process and the lack of memory for the inter-claim times, the reserve at time $t$ is equal to

$$
R(t)=\mathbb{E}\left[\sum_{i=1}^{\widehat{N}(T-t)} e^{-\widehat{\delta} \widehat{T}_{i}} \widehat{Y}_{i}\right] .
$$

Using the properties of the conditional expectation and the independence of the sequences $\left\{\widehat{\xi}_{i}, i \geq 1\right\}$ and $\left\{\widehat{Y}_{i}, i \geq 1\right\}$ we arrive at

$$
\begin{aligned}
R(t) & =\sum_{n=0}^{\infty} \mathbb{E}\left[\sum_{i=1}^{\widehat{N}(T-t)} e^{-\widehat{\delta} \widehat{T}_{i}} \widehat{Y}_{i} \mid \widehat{N}(T-t)=n\right] \operatorname{Pr}(\widehat{N}(T-t)=n) \\
& =\widehat{\mu} \sum_{n=0}^{\infty} \mathbb{E}\left[\sum_{i=1}^{\hat{N}(T-t)} e^{-\widehat{\delta} \widehat{T}_{i}} \mid \widehat{N}(T-t)=n\right] \operatorname{Pr}(\widehat{N}(T-t)=n)
\end{aligned}
$$

From Lemma 2.1 it follows that

$$
\begin{aligned}
\mathbb{E}\left[\sum_{i=1}^{n} e^{-\widehat{\delta} \widehat{\delta}_{i}} \mid \widehat{N}(T-t)=n\right] & =n \int_{0}^{T-t} e^{-\widehat{\delta} s} \frac{1}{T-t} d s \\
& =n \frac{1-e^{-\widehat{\delta}(T-t)}}{\widehat{\delta}(T-t)} .
\end{aligned}
$$


Finally, at time $t$, the reserve for the outstanding liabilities is equal to

$$
R(t)=\frac{\widehat{\mu} \widehat{\lambda}}{\widehat{\delta}}\left(1-e^{-\widehat{\delta}(T-t)}\right), \quad 0 \leq t<T .
$$

The insurance company can choose the parameters $\widehat{\delta}, \widehat{\lambda}, \widehat{\mu}$ taking into account its own attitude towards insurance risk $(\widehat{\lambda}, \widehat{\mu})$ and investment risk $(\widehat{\delta})$, or there exist solvency regulations which require the insurer to set reserves for future payments on a specified basis. In actuarial literature, the market reserve, which is the market value of insurance liabilities, is gaining much interest. For the concept of no-arbitrage between insurance and reinsurance markets and the arbitrage-free pricing of non-life business see Jang and $\mathrm{Kr}$ vavych (2004) and Sondermann (1991).

We investigate the following insurer's wealth path dependent disutility optimization problem. The aim of the insurer is to find an investment strategy which minimizes the quadratic loss function

$$
\begin{aligned}
\mathbb{E}\left[\int _ { 0 } ^ { T } \left\{(R(s)-X(s))^{2}+\alpha(\right.\right. & R(s)-X(s)\} d s \\
& \left.+\beta\left(X(T)^{2}-\alpha X(T)\right) \mid X(0)=x_{0}\right] .
\end{aligned}
$$

The above quadratic loss function penalizes deviations of the insurance surplus process below the reserve for outstanding liabilities, and rewards deviations above the reserve, which prompts the insurer to gain from the financial market. It is clear that the insurance company should have interest in keeping the wealth arising from the policy above the required reserve, just for solvency reasons. However, if the wealth crosses the upper barrier, which is given by the parameter $\alpha \geq 0$, the loss function penalizes those deviations above again. This means that when the wealth becomes too large it is cautiously invested. Notice that the "real" target imposed by such a quadratic function is $R(t)+\alpha / 2$. The parameter $\alpha \geq 0$ can be interpreted as the insurer's attitude towards investment risk, as a higher value of $\alpha$ corresponds to a lower risk aversion (see Haberman and Vigna (2002)). Stability of the surplus and the necessity of redistribution of large profits to the insured can serve as another explanation for laying down the upper barrier. The parameter $\beta>0$ attaches a weight to the terminal cost. Applying the investment strategy which minimizes (2.16) the insurer should meet all the contractual payments and make a profit on the issued policy.

In Sections 3 and 4 we use the following lemma.

Lemma 2.2. The solutions to the ordinary differential equations

$$
\begin{gathered}
1+a^{\prime}(t)+\phi a(t)=0, \quad a(T)=\beta, \\
-2 R(t)-\alpha-2 \mu \lambda a(t)+b^{\prime}(t)+\varphi b(t)=0, \quad b(T)=-\alpha \beta,
\end{gathered}
$$


where $\phi, \varphi$ are constant parameters, are given by

$$
a(t)=\frac{1}{\phi}\left(e^{\phi(T-t)}-1\right)+\beta e^{\phi(T-t)}
$$

and

$$
\begin{aligned}
b(t)= & -\alpha \beta e^{\varphi(T-t)}-\frac{\alpha}{\varphi}\left(e^{\varphi(T-t)}-1\right)-2 \int_{t}^{T} R(s) e^{\varphi(s-t)} d s \\
& -2 \mu \lambda \int_{t}^{T} a(s) e^{\varphi(s-t)} d s \\
= & -\alpha \beta e^{\varphi(T-t)}-\left(e^{\varphi(T-t)}-1\right)\left(\frac{2 \widehat{\mu} \widehat{\lambda}}{\widehat{\delta} \varphi}+\frac{\alpha}{\varphi}-\frac{2 \mu \lambda}{\phi \varphi}\right) \\
& +\left(e^{\varphi(T-t)}-e^{-\widehat{\delta}(T-t)}\right) \frac{2 \widehat{\mu} \widehat{\lambda}}{\widehat{\delta}(\varphi+\widehat{\delta})} \\
& -\left(e^{\varphi(T-t)}-e^{\phi(T-t)}\right) \frac{2 \mu \lambda}{\phi(\varphi-\phi)}(1+\beta \phi) .
\end{aligned}
$$

Proof. Solve the equations using variation of constants or check by direct substitution.

REMARK 2.1. Notice that $a(t) \geq \beta>0$ and $b(t) \leq 0$ for all $t \in[0, T]$.

Let $(a(t), b(t)) \sim(\phi, \varphi)$ denote the functions which solve the ordinary differential equations from Lemma 2.2 with parameters $\phi, \varphi$. This notation is used in Sections 3 and 4.

REMARK 2.2. Notice that we solve the differential equations in Lemma 2.2 and calculate the reserve in (2.15) assuming non-zero parameters. If a parameter appears to be zero, an appropriate limit in the given formulas should be taken.

In the next section we solve our optimization problem.

3. The solution of the optimization problem. In this section we present the solution to the optimization problem without constraints on control variables. This means that we allow short selling of assets and borrowing from a bank account.

Let $V(t, x)$ denote the optimal value function for our optimization problem defined as

$$
\begin{aligned}
V(t, x)= & \inf _{\theta(\cdot) \in \mathbb{R}^{n}} \mathbb{E}\left[\int_{t}^{T}\left\{(R(s)-X(s))^{2}+\alpha(R(s)-X(s))\right\} d s\right. \\
& \left.+\beta\left(X(T)^{2}-\alpha X(T)\right) \mid X(t)=x\right], \quad 0 \leq t<T
\end{aligned}
$$


and $V(T, x)=\beta\left(x^{2}-\alpha x\right)$. We first derive the Hamilton-Jacobi-Bellman equation heuristically, based on the Dynamic Programming Principle, and then state the verification theorem.

We have for all $t, 0<t \leq T$,

$$
\begin{aligned}
V(t-, x)= & \inf _{\theta(t) \in \mathbb{R}^{n}}\left\{(R(t-)-x)^{2} d t+\alpha(R(t-)-x) d t\right. \\
& +\mathbb{E}[V(t, X(t)) \mid X(t-)=x]\} .
\end{aligned}
$$

Applying Itô's formula for Lévy-type stochastic integrals (see Applebaum (2004)), we arrive at

$$
\begin{aligned}
d V(t, X(t))= & \frac{\partial V}{\partial t}(t, X(t-)) d t \\
& +\frac{\partial V}{\partial x}(t, X(t-))\left\{X(t-) \theta(t)^{T} \pi+X(t-) r\right\} d t \\
& +\frac{1}{2} \frac{\partial^{2} V}{\partial x^{2}}(t, X(t-)) X(t-)^{2} \theta(t)^{T} \mathbb{Q} \theta(t) d t \\
& +\frac{\partial V}{\partial x}(t, X(t-)) X(t-) \theta(t)^{T} \Sigma d \mathbb{W}(t) \\
& +\int_{0}^{\infty}\{V(t, X(t-)-y)-V(t, X(t-))\} M(d t, d y) .
\end{aligned}
$$

Let $V_{t}=\frac{\partial V}{\partial t}(t, x), V_{x}=\frac{\partial V}{\partial x}(t, x), V_{x x}=\frac{\partial^{2} V}{\partial x^{2}}(t, x)$. The Hamilton-JacobiBellman equation for our problem is of the form

$$
\begin{aligned}
0= & (R(t)-x)^{2}+\alpha(R(t)-x)+V_{t}+V_{x} \\
& +\int_{0}^{\infty}\{V(t, x-y)-V(t, x)\} \lambda p(d y) \\
& +\inf _{\theta \in \mathbb{R}^{n}}\left\{V_{x} x \theta^{T} \pi+\frac{1}{2} V_{x x} x^{2} \theta^{T} \mathbb{Q} \theta\right\} .
\end{aligned}
$$

The optimal control at time $t, 0<t \leq T$, is given by

$$
\theta^{*}(t)=-\frac{V_{x}}{V_{x x} x} \mathbb{Q}^{-1} \pi
$$

Substituting this value into the Hamilton-Jacobi-Bellman equation, we arrive at the partial integro-differential equation which the optimal value function $V(t, x)$ must satisfy. We find that

$$
\begin{aligned}
0= & (R(t)-x)^{2}+\alpha(R(t)-x)+V_{t}+V_{x} x r-\frac{1}{2} \frac{V_{x}^{2}}{V_{x x}} \pi^{T} \mathbb{Q}^{-1} \pi \\
& +\lambda \int_{0}^{\infty}\{V(t, x-y)-V(t, x)\} p(d y),
\end{aligned}
$$

with the boundary condition $V(T, x)=\beta\left(x^{2}-\alpha x\right)$. 
As the loss function is quadratic in $x$, we are looking for a quadratic solution of the form $V(t, x)=a(t) x^{2}+b(t) x+c(t)$. Then

$$
\begin{aligned}
& V_{t}=a^{\prime}(t) x^{2}+b^{\prime}(t) x+c^{\prime}(t), \quad V_{x}=2 a(t) x+b(t), \quad V_{x x}=2 a(t), \\
& \lambda \int_{0}^{\infty}\{V(t, x-y)-V(t, x)\} p(d y)=-2 \mu \lambda a(t) x+\mu_{2} \lambda a(t)-\mu \lambda b(t) .
\end{aligned}
$$

Substituting (3.7) and (3.8) into (3.6) we arrive at

$$
\begin{aligned}
0= & R(t)-2 R(t) x+x^{2}+\alpha R(t)-\alpha x+a^{\prime}(t) x^{2}+b^{\prime}(t) x+c^{\prime}(t) \\
& +2 r a(t) x^{2}+r b(t) x-2 \mu \lambda a(t) x+\mu_{2} \lambda a(t)-\mu \lambda b(t) \\
& -\pi^{T} \mathbb{Q}^{-1} \pi a(t) x^{2}-\pi^{T} \mathbb{Q}^{-1} \pi b(t) x-\pi^{T} \mathbb{Q}^{-1} \pi \frac{b(t)^{2}}{4 a(t)} .
\end{aligned}
$$

The problem of finding the optimal value function $V(t, x)$ satisfying the partial integro-differential equation (3.6) is equivalent to finding functions $a(t), b(t), c(t)$ satisfying the ordinary differential equations

$$
\begin{aligned}
& 0=1+a^{\prime}(t)+\left(2 r-\pi^{T} \mathbb{Q}^{-1} \pi\right) a(t), \quad a(T)=\beta, \\
& 0=-2 R(t)-\alpha-2 \mu \lambda a(t)+b^{\prime}(t)+\left(r-\pi^{T} \mathbb{Q}^{-1} \pi\right) b(t), \\
& b(T)=-\alpha \beta, \\
& 0=c^{\prime}(t)+(1+\alpha) R(t)+\mu_{2} \lambda a(t)-\mu \lambda b(t)-\frac{b(t)^{2}}{4 a(t)} \pi^{T} \mathbb{Q}^{-1} \pi, \\
& c(T)=0 .
\end{aligned}
$$

As the optimal control depends only on the functions $a(t), b(t)$ we solve only the first two equations. It suffices to set $\phi=2 r-\pi^{T} \mathbb{Q}^{-1} \pi$ and $\varphi=$ $r-\pi^{T} \mathbb{Q}^{-1} \pi$ to recover our solutions from Lemma 2.2 .

THEOREM 3.1. If there exists a function $V(t, x) \in \mathcal{C}^{1,2}([0, T] \times \mathbb{R})$ satisfying the Hamilton-Jacobi-Bellman equation

$$
\begin{aligned}
0= & (R(t)-x)^{2}+\alpha(R(t)-x)+V_{t}+V_{x} x r \\
& +\int_{0}^{\infty}\{V(t, x-y)-V(t, x)\} \lambda p(d y) \\
& +\inf _{\theta \in \mathbb{R}^{n}}\left\{V_{x} x \theta^{T} \pi+\frac{1}{2} V_{x x} x^{2} \theta^{T} \mathbb{Q} \theta\right\},
\end{aligned}
$$

with the boundary condition $V(T, x)=\beta\left(x^{2}-\alpha x\right)$, such that the processes

$$
\begin{gathered}
\int_{0}^{t} \frac{\partial V}{\partial x}(s, X(s-)) X(s-) \theta_{i}(s) d W_{j}(s), \quad i, j=1, \ldots, n, \\
\int_{0}^{t} \int_{0}^{\infty}\{V(s, X(s-)-y)-V(s, X(s-))\}(M(d s, d y)-\lambda p(d y) d s),
\end{gathered}
$$


are martingales for $t \in[0, T]$, and there exists an admissible control $\theta^{*}(\cdot)$ for which the infimum is reached, then

$$
\begin{aligned}
V(t, x)= & \inf _{\theta(\cdot) \in \mathbb{R}^{n}} \mathbb{E}\left[\int _ { t } ^ { T } \left\{(R(s)-X(s))^{2}+\alpha(R(s)-X(s)\} d s\right.\right. \\
& \left.+\beta\left(X(T)^{2}-\alpha X(T)\right) \mid X(t)=x\right],
\end{aligned}
$$

and $\theta^{*}(\cdot)$ is the optimal control for the problem.

REMARK 3.1. In order to have the martingale property for the processes (3.14) and (3.15), the function $V(t, x)$ should satisfy the conditions

$$
\begin{aligned}
& \mathbb{E}\left[\int_{0}^{T}\left\{\frac{\partial V}{\partial x}(t, X(t-)) X(t-) \theta_{i}(t)\right\}^{2} d t\right]<\infty, \quad i=1, \ldots, n, \\
& \mathbb{E}\left[\int_{0}^{T} \int_{0}^{\infty}\{V(t, X(t-)-y)-V(t, X(t-))\}^{2} \lambda p(d y) d t\right]<\infty .
\end{aligned}
$$

Proof of Theorem 3.1. The function satisfying (3.13) is of the form $a(t) x^{2}$ $+b(t) x+c(t)$, where $a(t), b(t), c(t)$ solve (3.10)-(3.12), and it is of class $\mathcal{C}^{1,2}([0, T] \times \mathbb{R})$. As $a(t)$ is positive for $0 \leq t \leq T$ and the matrix $\mathbb{Q}$ is positive definite, the feedback control

$$
\theta^{*}(t)=-\left(X^{*}(t-)+\frac{b(t)}{2 a(t)}\right) \frac{1}{X^{*}(t-)} \mathbb{Q}^{-1} \pi,
$$

where

$$
(a(t), b(t)) \sim\left(2 r-\pi^{T} \mathbb{Q}^{-1} \pi, r-\pi^{T} \mathbb{Q}^{-1} \pi\right),
$$

minimizes the quadratic form as required. One can also show that $\sup _{t \in[0, T]} \mathbb{E}\left|X^{*}(t)\right|^{4}<\infty$ (see the next section for a short explanation). It is straightforward to check that the control is a predictable process and satisfies (2.6) and (2.7), so it is admissible, and that conditions (3.17) and (3.18) are satisfied. Applying Itô's calculus, one can show that the derived function is the optimal value function for our optimization problem and the derived optimal control is the optimal investment strategy.

Next, we investigate some properties of the optimal investment strategy and the evolution of the insurer's wealth under this optimal strategy.

4. The optimal strategy and the insurer's wealth. The optimal investment strategy, at time $t, 0<t \leq T$, for our optimization problem without constraints on control variables is given in the feedback form

$$
\theta^{*}(t)=\left(g(t)-X^{*}(t-)\right) \frac{1}{X^{*}(t-)} \mathbb{Q}^{-1} \pi,
$$


where $g(t)=-b(t) / 2 a(t)$ and $(a(t), b(t)) \sim\left(2 r-\pi^{T} \mathbb{Q}^{-1} \pi, r-\pi^{T} \mathbb{Q}^{-1} \pi\right)$. The evolution of the insurer's wealth under the optimal investment strategy is given by

$$
\begin{aligned}
d X^{*}(t)= & X^{*}(t-) \theta^{*}(t)^{T} \pi d t+X^{*}(t-) \theta^{*}(t)^{T} \Sigma d \mathbb{W}(t) \\
& +X^{*}(t-) r d t-d J(t), \quad X^{*}(0)=x_{0} .
\end{aligned}
$$

Substituting the optimal strategy we arrive at

$$
\begin{aligned}
d X^{*}(t)= & \left(g(t)-X^{*}(t-)\right) \pi^{T} \mathbb{Q}^{-1} \pi d t+X^{*}(t-) r d t \\
& +\left(g(t)-X^{*}(t-)\right)\left(\Sigma^{-1} \pi\right)^{T} d \mathbb{W}(t)-d J(t), \quad X^{*}(0)=x_{0}
\end{aligned}
$$

As the coefficients in the diffusion part of the equation (4.3) satisfy Lipschitz and growth conditions and the compound Poisson distribution has finite fourth moment, the above stochastic differential equation has a unique solution such that $\sup _{t \in[0, T]} \mathbb{E}\left|X^{*}(t)\right|^{4}<\infty$.

We solve this stochastic differential equation. Let $H(t)=g(t)-X^{*}(t)$. The stochastic differential of the process $\{H(t), 0 \leq t \leq T\}$ is

$$
\begin{aligned}
d H(t)= & g^{\prime}(t) d t-d X^{*}(t) \\
= & g^{\prime}(t) d t-g(t) r d t+H(t-)\left(r-\pi^{T} \mathbb{Q}^{-1} \pi\right) d t \\
& -H(t-)\left(\Sigma^{-1} \pi\right)^{T} d \mathbb{W}(t)+d J(t), \quad H(0)=g(0)-x_{0} .
\end{aligned}
$$

Let us introduce the process $\{Z(t), 0 \leq t \leq T\}$, with continuous sample paths, of the form

$$
Z(t)=e^{-\left(r-\pi^{T} \mathbb{Q}^{-1} \pi\right) t+\frac{1}{2}\left\|\Sigma^{-1} \pi\right\|^{2} t+\left(\Sigma^{-1} \pi\right)^{T} \mathbb{W}(t)},
$$

where $\|\cdot\|$ denotes the vector norm. The stochastic differential of this process is

$$
d Z(t)=Z(t)\left\{-\left(r-\pi^{T} \mathbb{Q}^{-1} \pi\right) d t+\left\|\Sigma^{-1} \pi\right\|^{2} d t+\left(\Sigma^{-1} \pi\right)^{T} d \mathbb{W}(t)\right\}
$$

Multiplying both sides of (4.4) by $Z(t)$ we arrive at

$$
\begin{array}{r}
Z(t) d H(t)-H(t-) Z(t)\left(r-\pi^{T} \mathbb{Q}^{-1} \pi\right) d t+H(t-) Z(t)\left(\Sigma^{-1} \pi\right)^{T} d \mathbb{W}(t) \\
=Z(t)\left(g^{\prime}(t) d t-g(t) r d t+d J(t)\right), \quad H(0)=g(0)-x_{0} .
\end{array}
$$

Let us recall Itô's product formula for two Lévy-type stochastic integrals $Y_{1}(t)$ and $Y_{2}(t)$ (see Applebaum (2004)):

$$
d\left(Y_{1}(t) Y_{2}(t)\right)=Y_{1}(t-) d Y_{2}(t)+Y_{2}(t-) d Y_{1}(t)+d\left[Y_{1}, Y_{2}\right] d t
$$

where

$$
\left[Y_{1}, Y_{2}\right](t)=\left[Y_{1}^{c}, Y_{2}^{c}\right](t)+\sum_{0 \leq s \leq t} \triangle Y_{1}(s) \triangle Y_{2}(s),
$$

$[\cdot](t)$ denotes the quadratic variation process and $c$ denotes the continuous parts of the processes. Now we recognize that the left-hand side of (4.7) is the 
stochastic differential for $H(t) Z(t)$. Notice that $Z(t)$ has continuous sample paths and the second term in (4.9) is zero. We arrive at

$$
\begin{aligned}
H(t)= & \frac{1}{Z(t)}\left\{H(0)+\int_{0}^{t} Z(s)\left(g^{\prime}(s)-g(s) r\right) d s\right. \\
& \left.+\int_{0}^{t} \int_{0}^{\infty} Z(s) y M(d s, d y)\right\},
\end{aligned}
$$

where the last term is a Poisson stochastic integral. Finally, the insurer's wealth, under the optimal investment strategy, at time $t$ is equal to

$$
\begin{aligned}
X^{*}(t)= & g(t)-\frac{1}{Z(t)}\left\{g(0)-x_{0}+\int_{0}^{t} Z(s)\left(g^{\prime}(s)-g(s) r\right) d s\right. \\
& \left.+\int_{0}^{t} \int_{0}^{\infty} Z(s) y M(d s, d y)\right\} .
\end{aligned}
$$

It is possible to derive a formula for the expected value of the insurer's wealth under the optimal investment strategy. If we take the expected value on both sides of $(4.3)$ and let $m(t)=\mathbb{E} X^{*}(t)$, then we obtain an ordinary differential equation for the function $m(t)$ :

$$
m^{\prime}(t)=(g(t)-m(t)) \pi^{T} \mathbb{Q}^{-1} \pi+m(t) r-\lambda \mu, \quad m(0)=x_{0} .
$$

This equation has the solution

$$
\begin{aligned}
m(t)= & x_{0} e^{\left(r-\pi^{T} \mathbb{Q}^{-1} \pi\right) t}-\frac{\lambda \mu}{r-\pi^{T} \mathbb{Q}^{-1} \pi}\left\{e^{\left(r-\pi^{T} \mathbb{Q}^{-1} \pi\right) t}-1\right\} \\
& +\pi^{T} \mathbb{Q}^{-1} \pi \int_{0}^{t} g(s) e^{\left(r-\pi^{T} \mathbb{Q}^{-1} \pi\right)(t-s)} d s .
\end{aligned}
$$

The insurance company can choose the parameters $\widehat{\delta}, \widehat{\lambda}, \widehat{\mu}, \alpha$ and $\beta$ in the optimization process. These parameters reflect the insurer's attitude towards the risk it faces and introduce the risk profile of the insurer.

Let us consider the case of one risky asset. Then the optimal investment strategy is given by

$$
\theta^{*}(t)=\left(g(t)-X^{*}(t-)\right) \frac{1}{X^{*}(t-)} \frac{a-r}{\sigma^{2}},
$$

where

$$
g(t)=-\frac{b(t)}{2 a(t)} \quad \text { and } \quad(a(t), b(t)) \sim\left(2 r-\left(\frac{a-r}{\sigma}\right)^{2}, r-\left(\frac{a-r}{\sigma}\right)^{2}\right) .
$$

The parameters $\widehat{\delta}, \widehat{\lambda}, \widehat{\mu}$ have an influence on the reserve. The higher the loss frequency $\hat{\lambda}$, the higher the reserve, and the same relation holds for the 
expected severity of claims $\widehat{\mu}$. However, the lower the rate of return $\widehat{\delta}$, the higher the reserve. The reserve constitutes the lower and upper barrier on the loss function and has an impact on the optimal investment strategy and on the insurer's expected wealth under this strategy. The higher the reserve, the higher the fraction of the wealth invested in the risky asset (given the same positive level of available wealth) and the higher the expected value of the insurer's wealth. This can be seen from equations (2.20), (4.13) and (4.14), as $g(t)$ is decreasing in $b(t)$ and $b(t)$ is decreasing in the reserve.

The parameter $\alpha$ affects the "real" target imposed by the loss function and it also has an impact on the optimal investment strategy and on the insurer's expected wealth under this strategy. The higher the value of $\alpha$, the higher the fraction of the wealth invested in the risky asset (given the same positive level of available wealth) and the higher the expected value of the insurer's wealth. This can be seen again from equations (2.20), (4.13) and (4.14), as $b(t)$ is decreasing in $\alpha$. The above relations are intuitively clear. Notice that the statements concerning the insurer's expected wealth also hold in the multi-asset economy, as $\pi^{T} \mathbb{Q}^{-1} \pi$ is positive (the matrix $\mathbb{Q}^{-1}$ is positive definite).

It is straightforward to derive regions where the strategy of short selling the asset or borrowing from a bank account is optimal. We observe that

$$
\begin{aligned}
& \theta^{*}(t)<0 \Leftrightarrow X^{*}(t-)<0 \vee X^{*}(t-)>g(t), \\
& \theta^{*}(t)>1 \Leftrightarrow 0<X^{*}(t-)<\frac{g(t)}{1+\frac{\sigma^{2}}{a-r}} .
\end{aligned}
$$

If the wealth is low (but positive), the insurer should borrow from a bank account and invest aggressively in the risky asset in order to reach the desired target. When the wealth is increasing, the insurer should invest more cautiously and decrease the fraction of the wealth invested in the risky asset. However, if the wealth is too large, then the optimal strategy is short-selling of the asset, which means throwing money away. Notice that in the optimization problem with constraints on control variables (no short selling of assets or borrowing from a bank account), $\alpha$ would be the parameter setting the amount of wealth which "satisfies" the insurer and over which the insurer takes no investment risk and invests only in the risk free asset. In the case of negative wealth, the short-selling strategy gives the insurer a chance to recover the positive level of wealth. This coincides with the properties of the applied quadratic loss function.

Summing up, in this paper we have investigated the construction of an investment strategy for a non-life insurance company. We have dealt with a wealth path dependent disutility optimization problem and for a quadratic loss function we have derived an optimal investment strategy. The solution 
can easily be implemented in practice.

\section{References}

A. Ahcan, G. Darkiewicz, J. Dhaene, M. Goovaerts and T. Hoedemakers (2004), Optimal portofolio selection: Applications in insurance bussiness, Report, Catholic Univ. of Leuven.

D. Applebaum (2004), Lévy Processes and Stochastic Calculus, Cambridge Univ. Press.

S. Browne (1995), Optimal investment policies for a firm with a random risk process: exponential utility and minimizing of the probability of ruin, Math. Oper. Res. 20, 937-957.

A. J. G. Cairns (2000), Some notes on the dynamics and optimal control of stochastic pension fund models in continuous time, ASTIN Bull. 30, 19-55.

S. Haberman and E. Vigna (2002), Optimal investment strategies and risk measures in defined contribution pension schemes, Insurance Math. Econom. 31, 35-69.

C. Hipp (2002), Stochastic control with applications in insurance, Report, Univ. of Karlsruhe.

C. Hipp and M. Plum (2000), Optimal investment for insurers, Insurance Math. Econom. $26,215-228$.

C. Hipp and M. Vogt (2003), Optimal dynamic XL reinsurance, ASTIN Bull. 33, 193-208.

B. Højgaard and M. Taksar (2000), Optimal risk control for a large corporation in the presence of returns on investments, Finance Stoch. 5, 527-547.

F. Hubalak and W. Schachermayer (2004), Optimizing expected utility of dividend payments for a Brownian risk process and a peculiar nonlinear ODE, Insurance Math. Econom. 34, 193-225.

J. Jang and Y. Krvavych (2004), Arbitrage-free premium calculation for extreme losses using the shot noise process and the Esscher transform, ibid. 35, 97-111.

R. Korn (2005), Worst-case scenario investment for insurers, ibid. 36, 1-11.

J. Paulsen (2003), Optimal dividend payouts for diffusions with solvency constraints, Finance Stoch. 4, 457-474.

K. I. Sato (1999), Lévy Processes and Infinitely Divisible Distributions, Cambridge Univ. Press.

H. Schmidli (2002), On minimising the ruin probability by investment and reinsurance, Ann. Appl. Probab. 12, 890-907.

D. Sondermann (1991), Reinsurance in arbitrage-free market, Insurance Math. Econom. 10, 191-202.

Division of Probabilistic Methods

Institute of Econometrics

Warsaw School of Economics

Al. Niepodległości 162

02-554 Warszawa, Poland

E-mail: lukasz.delong@sgh.waw.pl

Received on 20.12.2004;

revised version on 14.6.2005 